\title{
Quantum Knizhnik-Zamolodchikov equation, Totally Symmetric Self-Complementary Plane Partitions and Alternating Sign Matrices
}

\author{
P. Zinn-Justin * \\ P. Di Francesco
}

\begin{abstract}
We present multiresidue integral formulae for partial sums in the basis of link patterns of the polynomial solution to the level $1 U_{q}(\widehat{\mathfrak{s l}})$ quantum Knizhnik-Zamolodchikov equation at generic values of the quantum parameter $q$. These allow for rewriting and generalizing a recent conjecture [Di Francesco '06] connecting the above to generating polynomials for weighted Totally Symmetric Self-Complementary Plane Partitions. We reduce the corresponding conjectures to a single integral identity.
\end{abstract}

$03 / 2007$

* Laboratoire de Physique Théorique et Modèles Statistiques (CNRS, UMR 8626); Univ ParisSud, Orsay, F-91405.

$\diamond$ Service de Physique Théorique de Saclay, CEA/DSM/SPhT, URA 2306 du CNRS, F-91191 Gif sur Yvette Cedex. 


\section{Introduction}

In the past few years, we have witnessed the development of an ever increasingly complex set of interrelations betwen various combinatorial objects and some integrable models: on the one hand, Alternating Sign Matrices, Fully Packed Loops and Plane Partitions; on the other hand, XXZ and related spin chains, and lattice loop models. These will be reviewed below, the goal of this paper being to provide some tools to prove the combinatorial conjectures that were formulated, and in particular the conjecture of [1]. We believe that the tools developed here may apply to a number of other situations as well, for instance to the case of different boundary conditions for the loop model (e.g. open boundary case, see conjectures in [2]).

\subsection{Integrable loop model and the Razumov-Stroganov conjecture}

The Temperley-Lieb model of loops is defined on a semi-infinite cylinder of square lattice, with even perimeter $2 n$ whose edge centers are labelled $1,2, \ldots, 2 n$ counterclockwise. The configurations of the model are obtained by picking any of the two possible face configurations $\square$ or $\square$ at each face of the lattice. We see that the configurations of the model form either closed loops or open curves joining boundary points by pairs, without any intersection between curves. In fact, each configuration realizes a planar pairing of the boundary points via a link pattern, namely a diagram in which $2 n$ labelled and regularly spaced points of a circle are connected by pairs via non-intersecting straight segments. The set of link patterns over $2 n$ points is denoted by $\mathcal{L}_{2 n}$, and its cardinality is $c_{n}=(2 n) ! /(n !(n+1) !)$. We also view $\pi \in \mathcal{L}_{2 n}$ as an involution of $\mathcal{S}_{2 n}$ without fixed points.

We moreover consider an inhomogeneous model in which probabilities are associated to these face configurations depending on the row $i$ at which they are inserted. We use the following parameterization of the probabilities in terms of variables $z_{i}$ :

$$
T_{n}\left(t ; z_{1}, \ldots, z_{2 n}\right)=\prod_{i=1}^{2 n}\left(\frac{q z_{i}-q^{-1} t}{q t-q^{-1} z_{i}} \square+\frac{z_{i}-t}{q t-q^{-1} z_{i}} \square\right)
$$

where $T$ is the transfer matrix that adds one row to the semi-infinite cylinder, and here $q=$

$\mathrm{e}^{2 i \pi / 3} . t$ is an additional variable which can be absorbed into the $z_{i}$; however it is useful to introduce it because of the commutation relations (due to integrability) $\left[T_{n}(t), T_{n}\left(t^{\prime}\right)\right]=0$ at $z_{i}$ fixed. 
We may now ask what is the probability $P_{\pi}\left(z_{1}, \ldots, z_{2 n}\right)$ in random configurations of the model that the boundary points be pair-connected according to a given link pattern $\pi \in \mathcal{L}_{2 n}$. Forming the vector $P_{n}\left(z_{1}, \ldots, z_{2 n}\right)$ whose components are the $P_{\pi}\left(z_{1}, \ldots, z_{2 n}\right)$ in a vector space with canonical basis indexed by link patterns $\pi$, we immediately see that it satisfies the eigenvector condition

$$
T_{n}\left(t ; z_{1}, \ldots, z_{2 n}\right) P_{n}\left(z_{1}, \ldots, z_{2 n}\right)=P_{n}\left(z_{1}, \ldots, z_{2 n}\right)
$$

Eq. (1.2) does not specify the normalization of $P_{n}$, which is given by the fact that the total probability is 1 . It is however more convenient to consider another normalization of the solution of Eq. (1.2), which we denote by $\Psi_{n}$, and such that its components $\Psi_{\pi}$ are coprime polynomials in the variables $z_{i}$. This only leaves a numerical multiplicative constant to be fixed later.

The main conjectures concern the homogeneous limit $z_{1}=\cdots=z_{2 n}=1$. In [3], it was noticed that:

(i) the $\Psi_{\pi} / \Psi_{\pi_{0}}$ are positive integers, where $\Psi_{\pi_{0}}$ is the smallest component;

(ii) $\Psi_{\max } / \Psi_{\pi_{0}}=A_{n-1}$, where $\Psi_{\max }$ is the largest component, and

(iii) $\sum_{\pi \in \mathcal{L}_{2 n}} \Psi_{\pi} / \Psi_{\pi_{0}}=A_{n}$,

where $A_{n}$ is the number of Alternating Sign Matrices (ASMs) of size $n$ (see the book by Bressoud [4] for a complete saga and references):

$$
A_{n}=\frac{1 ! 4 ! 7 ! \cdots(3 n-2) !}{n !(n+1) !(n+2) ! \cdots(2 n-1) !}
$$

$A_{n}$ is also the number of Totally Symmetric Self-Complementary Plane Partitions (TSSCPPs), which may be viewed as the tiling configurations of a regular hexagon of edge size $2 n$ drawn on the triangular lattice, by means of elementary rhombi, and enjoying all possible symmetries of the hexagon. Until recently, this fact did not seem particularly relevant to this model, especially in view of the Razumov-Stroganov (RS) conjecture [5]. The latter claims that $\Psi_{\pi} / \Psi_{\pi_{0}}$ is the number of Fully Packed Loops configurations (FPL) with connectivity $\pi$. The latter are configurations of loops drawn on the edges of a square grid of size $n \times n$, such that at each vertex exactly two edges are occupied by loop edges, and with the boundary condition that every second edge of the boundary is occupied. Labelling the latter $1,2, \ldots, 2 n$ allows for keeping track of pairings of boundary edges via curves, hence to associate to each link pattern $\pi$ a set of FPL configurations. Due to their definition, the FPLs are in simple bijection with the configurations of the six-vertex model 
with domain-wall boundary conditions, as well as with ASMs. The RS conjecture therefore gives an enumerative interpretation of points (i) and (iii) (and in principle also of (ii)). Note that on the contrary, no bijection is known betteen ASMs and TSSCPPs.

Point (iii) was proved in [6] via its generalization to the inhomogeneous model, which reads $\sum_{\pi} \Psi_{\pi}=Z_{n}\left(z_{1}, \ldots, z_{2 n}\right)$, where $Z_{n}$ is the Izergin-Korepin partition function of the six-vertex model with domain-wall boundary conditions [7], which already appeared in [8] in the context of ASM enumeration.

\subsection{TSSCPP conjecture and the minimal polynomial solutions to the qKZ equation}

It was noted in [6] that Eq. [1.2] may be solved by writing instead the consequences on $\Psi_{n}$ of the intertwining relations for the transfer matrix (1.1) of the inhomogeneous loop model. The latter could eventually be reexpressed as the level $1 U_{q}(\widehat{\mathfrak{s l}})$ quantum KnizhnikZamolodchikov ( $q \mathrm{KZ}$ ) equation [9] for the groundstate vector $\Psi_{n}$, at $q=-e^{i \pi / 3}$. When written in components, this equation allows for expressing all polynomial components $\Psi_{\pi}$ in terms of $\Psi_{\pi_{0}}$, in a triangular manner. $\Psi_{\pi_{0}}$ is then determined as the minimal degree polynomial subject to all divisibility properties inherited from the intertwining relations, and has a nice factorized form. The $q \mathrm{KZ}$ equation satisfied by $\Psi_{n}$ may also alternatively be interpreted by stating that the components $\Psi_{\pi}$ form a polynomial representation of the affine Temperley-Lieb algebra, including a weight $\tau=-q-q^{-1}=1$ per loop.

There is a natural $q$-deformation of the problem obtained either by looking for polynomial representations of the affine Temperley-Lieb algebra with weight $\tau=-q-q^{-1}$ per loop [10] or equivalently in the form of the polynomial solutions of the generic $q$ level $1 U_{q}(\widehat{\mathfrak{s l}}) q \mathrm{KZ}$ equation [11]. It was noted in [11] and [12] that the properly normalized vector $\Psi_{n}$ displays nice combinatorial properties in the homogeneous limit, but now as a function of $\tau$. It was indeed noted that each ratio $\Psi_{\pi} / \Psi_{\pi_{0}}$, when expressed at $z_{i}=1$, appears to be a polynomial of $\tau$ with non-negative integer coefficients. It would of course have been tempting to infer the existence of a $q$-deformed RS-type conjecture, in which these quantities could be interpreted as weighted sums of FPL configurations with fixed connectivities.

A first step was made in [1] in this direction, by proposing a conjecture for the sum of all components as a polynomial of $\tau$. But surprisingly, the right combinatorial object conjecturally underlying this sum is not the FPLs or ASMs, but rather the TSSCPPs.

1 Actually, some earlier works 13 had already unearthed some connections between the loop model in slightly deformed cylinder geometries and refined TSSCPP counting, but at $\tau=1$. 
To understand the conjecture, it is first necessary to express the TSSCPPs as sets of non-intersecting lattice paths (NILPs) by noting that tiles in a fundamental domain of the hexagon may be organized into sets of non-intersecting broken lines propagating within that region. Once this is done, the TSSCPPs may be viewed as configurations of lattice paths which may have two possible types of steps, say vertical and diagonal when represented on a square lattice.

The TSSCPP conjecture states that

(iv) $\sum_{\pi} \Psi_{\pi} / \Psi_{\pi_{0}}$ is the generating polynomial for TSSCPPs of size $2 n$ with a weight $\tau$ per vertical step in their NILP formulation.

Note that point (iv) reduces to point (iii) at $\tau=1$. The main purpose of this paper is to address the TSSCPP conjecture (iv).

It is interesting to note that refinements of TSSCPPs have been already related to refined enumerations of ASMs (c.f. [14]), but these concern always "boundary" weightings of configurations (e.g. weighting only the last or first step of the TSSCPPs, versus keeping track of the positions of 1's in the first row or column of the ASMs). Here we rather have a "bulk" weighting, that does not distinguish the boundary steps. The question of finding a good interpretation for the $\tau$ weighting in ASMs or FPLs remains open, and might shed some light on a possible ASM-TSSCPP bijection.

\subsection{Plan}

In this paper, we first address the weighted enumeration of TSSCPPs (Section 2). The results are derived by use of the formulation of the latter in terms of non-intersecting lattice paths (NILPs), and the celebrated Lindström-Gessel-Viennot (LGV) determinantal formula [15], and take the form of multiple contour (residue) integrals.

In a second step (Section 3), we shall write the space of polynomial solutions of the $q \mathrm{KZ}$ equation in terms of multiple contour (residue) integrals, by using a new set of vectors indexed by sequences of integers (distinct from the so-called spin basis used in the language of the corresponding spin chains). Upon going to the loop (link pattern) basis, this will give us integral formulae for partial sums of components of $\Psi$. In particular, we will get

integral formulae for the maximal component $\Psi_{\max }$ as well as for the sum of all components $\sum_{\pi} \Psi_{\pi}$. The details of the change of basis from this new set of vectors to link patterns is given in appendix A, while the relation to spin basis components is detailed in appendix B.

We then show in Section 4 how the TSSCPP conjecture (iv) as well as (ii) and (iii) above are all consequences of a simple multiresidue integral identity, whose proof is left to future work. 


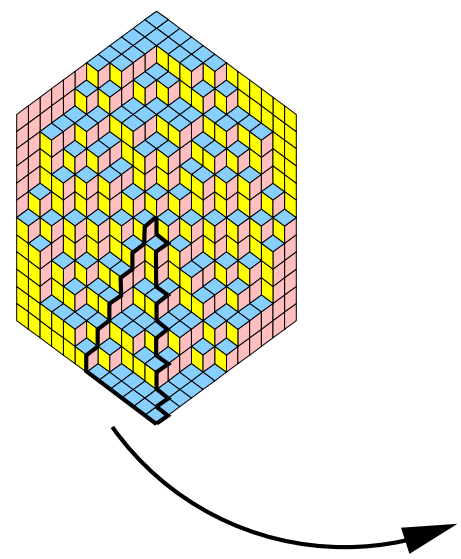

TSSCPP
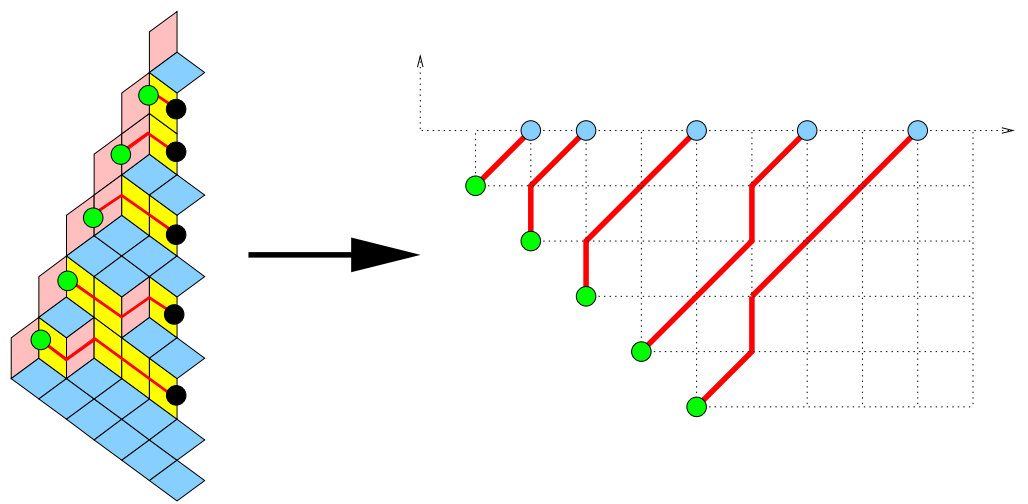

Fig. 1: From TSSCPP to NILP. Including a weight $t_{i}$ per vertical step in the $i$-th horizontal slice from top, the NILP configuration receives the weight $t_{2}^{2} t_{3} t_{4}$.

\section{TSSCPP}

\subsection{Definitions}

Totally Symmetric Self-Complementary Plane Partitions are rhombus tilings of a regular hexagon of the triangular lattice, of size $2 n \times 2 n \times 2 n$, enjoying rotational and reflection symmetries of the hexagon, implementing the self-complementation property that, when viewed as the $(1,1,1)$ direction perspective view of a pile of elementary cubes in the positive quadrant of $\mathbb{Z}^{3}$, the complement to the pile within the cube of size $2 n$ is an identical copy of it.

This allows to restrict TSSCPPs to a fundamental domain made of $1 / 12$ th of the original hexagon (see Fig.1), and to count them using Non-Intersecting Lattice Paths (NILPs). The latter are simply obtained by following successions of two of the three types of tiles used in the tiling of the fundamental domain. Deforming slightly the geometry, we arrive at the problem of counting $n-1$ NILPs on the square lattice, each path taking vertical steps of $(0,1)$ or diagonal steps of $(1,1)$ only, the $i$-th path starting at point $(i,-i)$ and ending up on the line $y=0$, after $i$ steps. This leads to the total number of such paths $N_{10}(2 n)$, as given for instance by a Lindström-Gessel-Viennot (LGV) type formula [15], expressing the number of NILPs with fixed endpoints as the determinant of the numbers of paths from the $i$-th starting point to the $j$-th endpoint say $\left(r_{j}, 0\right)$, and summed over the positions of the endpoints. In Ref.[1], a refinement of these numbers was introduced 
via the generating polynomial $N_{10}(2 n \mid \tau)$ of TSSCPPs counted with a weight $\tau$ per vertical step, and given by the following LGV type formula:

$$
N_{10}(2 n \mid \tau)=\sum_{\substack{1 \leq r_{1}<r_{2}<\cdots<r_{n-1} \\
r_{i} \leq 2 i}} \operatorname{det}_{1 \leq i, j \leq n-1}\left(\left(\begin{array}{c}
i \\
r_{j}-i
\end{array}\right) \tau^{2 i-r_{j}}\right)
$$

ExAMPLE. For $n=3$, there are 7 TSSCPP configurations:

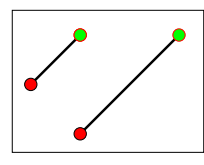

1

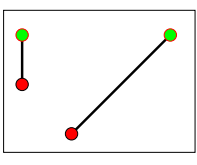

$\tau$

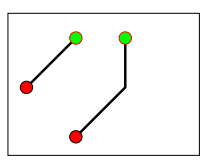

$\tau$

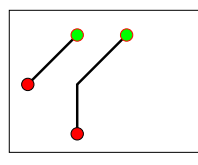

$\tau$

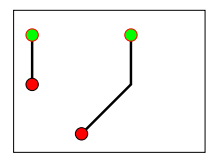

$\tau^{2}$

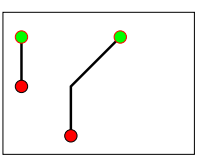

$\tau^{2}$



$\tau^{3}$

resulting in the polynomial $N_{10}(6 \mid \tau)=1+3 \tau+2 \tau^{2}+\tau^{3}$.

\subsection{Integral formulae}

Let us derive a simple multiple contour integral formula for the generating polynomial $N_{10}\left(t_{1}, t_{2}, \ldots t_{n-1}\right)$ of TSSCPPs in their NILP formulation with a weight $t_{i}$ per vertical step in the $i$-th slice delimited by the lines $y=1-i$ and $y=-i$. We use again the LGV formula, including the weighting of paths by the $t_{i}$ 's. The formula reads

$$
N_{10}\left(t_{1}, t_{2}, \ldots t_{n-1}\right)=\sum_{\substack{1 \leq r_{1}<r_{2}<\cdots<r_{n-1} \\ r_{i} \leq 2 i}} \operatorname{det}_{1 \leq i, j \leq n-1} \mathcal{P}_{i, r_{j}}
$$

where $\mathcal{P}_{i, r}$ is the weighted sum over all lattice paths from $(i,-i)$ to $(r, 0)$. Such a path has $r-i$ diagonal steps and $2 i-r$ vertical ones, to be taken within the $i$ first slices, hence

$$
\mathcal{P}_{i, r}=\sum_{1 \leq i_{1}<i_{2}<\cdots<i_{2 i-r}} \prod_{\ell=1}^{2 i-r} t_{i_{\ell}}=\left.\prod_{k=1}^{i}\left(1+t_{k} u\right)\right|_{u^{2 i-r}}
$$

where the subscript stands for the coefficient of the corresponding power of $u$ in the polynomial. Introducing an extra trivial path starting and ending at the origin, such that $\mathcal{P}_{i, 0}=\delta_{i, 0}$ and substituting 2.3 into $(2.2$, we get the following coefficient identity:

$$
N_{10}\left(t_{1}, t_{2}, \ldots t_{n-1}\right)=\left.\prod_{1 \leq i<j \leq n} \frac{\left(u_{j}-u_{i}\right)\left(1+t_{i} u_{j}\right)}{1-u_{i} u_{j}} \prod_{i=1}^{n} \frac{1}{1-u_{i}}\right|_{\prod_{i=1}^{n} u_{i}^{2 i-2}}
$$


where again the subscript stands for the coefficient of the corresponding monomial in the power series expansion of the rational fraction around 0. To prove [2.4), one uses the multilinearity of the determinant to rewrite 22.2$](2.3)$ as

$$
N_{10}\left(t_{1}, t_{2}, \ldots t_{n-1}\right)=\prod_{i=1}^{n} \oint \frac{\mathrm{d} u_{i}}{u_{i}^{2 i-1}} \prod_{k=1}^{i-1}\left(1+t_{k} u_{i}\right) \sum_{0 \leq r_{0}<r_{1}<r_{2}<\cdots<r_{n-1}} \operatorname{det}_{1 \leq i, j \leq n}\left(u_{i}^{r_{j-1}}\right)
$$

where we have replaced the condition $r_{0}=0$ by a summation over $r_{0} \geq 0$, that does not alter the result of the integration. Finally, we evaluate the sum of determinants to be

$$
\sum_{0 \leq r_{0}<r_{1}<r_{2}<\cdots<r_{n-1}} \operatorname{det}_{1 \leq i, j \leq n}\left(u_{i}^{r_{j-1}}\right)=\prod_{1 \leq i<j \leq n} \frac{u_{j}-u_{i}}{1-u_{i} u_{j}} \prod_{i=1}^{n} \frac{1}{1-u_{i}}
$$

This is nothing but the sum of all Schur functions of the $n$ arguments $u_{1}, \ldots, u_{n}$, multiplied by the Vandermonde determinant. The result for this sum is standard (see for instance eq 4.17 of [4]). Eq. (2.4) then follows.

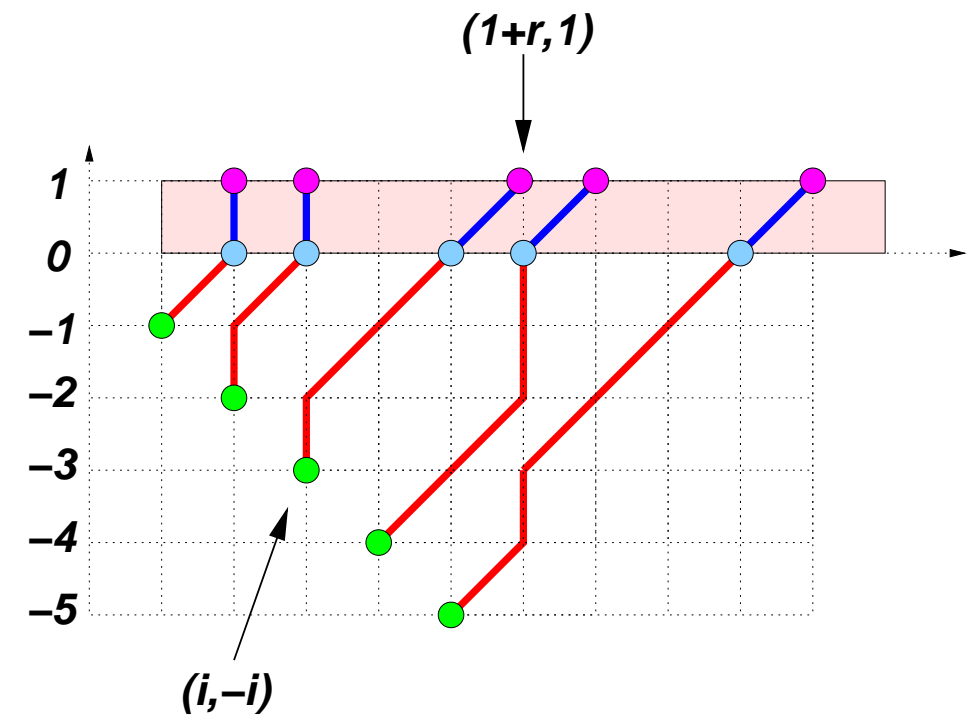

Fig. 2: NILPs for Modified TSSCPPs. We have added an extra top slice of steps to the original NILPs of Fig1, so that the new NILPs end up on the line $y=1$. The arrival points, of the form $\left(1+r_{i}, 1\right)$ are further constrained by imposing that all successive differences $r_{i+1}-r_{i}$ be odd integers. With this, there is exactly one way of completing a TSSCPP into a modified one, thus the two sets of objects are in bijection.

For later purposes, we are also interested in modified TSSCPPs defined as follows. We simply add up one extra step to all previous TSSCPPs in a top slice between $y=0$ 
and $y=1$, with a weight $t_{0}$ per vertical step in this extra slice, and further constrained as follows. We demand that the consecutive new endpoints, of the form $\left(r_{i}+1,1\right)$, differ only by some odd integers, namely $r_{i+1}-r_{i}$ odd for all $i$, and $r_{1}=1$. The reason for this is that there is a bijection between these and the TSSCPPs above. Indeed, the constraint on the new endpoints ensures that the restrictions of these modified TSSCPPs to the region below the $\mathrm{x}$ axis be in bijection with regular TSSCPPs. We denote by $N_{10}^{\prime}\left(t_{0}, t_{1}, \ldots, t_{n-1}\right)$ the corresponding generating polynomial.

EXAMPLE. The seven TSSCPPs at $n=3$ are augmented as follows:

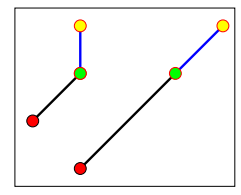

$\boldsymbol{t}_{0}$

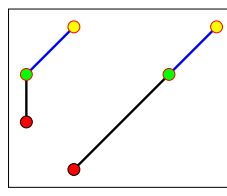

$t_{1}$

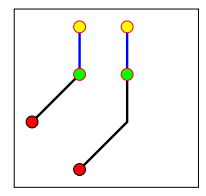

$t_{0}^{2} t_{1}$

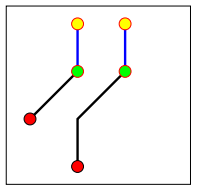

$t_{0}^{2} t_{2}$

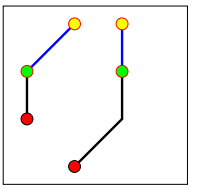

$t_{0} t_{1}^{2}$



$t_{0} t_{1} t_{2}$

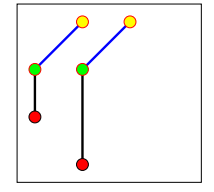

$t_{1}^{2} t_{2}$

and the corresponding generating polynomial reads $N_{10}^{\prime}\left(t_{0}, t_{1}, t_{2}\right)=\left(1+t_{0} t_{1}\right)\left(t_{0}+t_{1}\right)+$ $\left(t_{0}^{2}+t_{0} t_{1}+t_{1}^{2}\right) t_{2}$

We have:

$$
N_{10}^{\prime}\left(t_{0}, t_{1}, \ldots, t_{n-1}\right)=\left.\prod_{1 \leq i<j \leq n} \frac{\left(u_{j}-u_{i}\right)\left(1+t_{i} u_{j}\right)}{1-u_{i} u_{j}} \prod_{i=1}^{n} \frac{1+t_{0} u_{i}}{1-u_{i}^{2}}\right|_{\prod_{i=1}^{n} u_{i}^{2 i-2}}
$$

As expected from the above bijection, we recover [2.4) at $t_{0}=1$. Eq. [2.7] is proved in much the same way as $(2.4)$. Denoting by $\mathcal{Q}_{i, r}$ the weighted sum over paths from $(i,-i)$ to $(r+1,1)$, we now have

$$
\mathcal{Q}_{i, r}=\left.\prod_{k=0}^{i}\left(1+t_{k} u\right)\right|_{u^{2 i-r}}
$$

and, as in the previous case, adding up a trivial path from the origin to $(1,1)$ (hence an extra arrival point $r_{0}=0$ ), we have

$$
N_{10}^{\prime}\left(t_{0}, t_{1}, \ldots, t_{n-1}\right)=\prod_{i=1}^{n} \oint \frac{\mathrm{d} u_{i}}{2 \pi i u_{i}^{2 i-1}} \prod_{k=0}^{i}\left(1+t_{k} u_{i}\right) \sum_{\substack{0 \leq r_{0}<r_{1}<\cdots<r_{n-1} \\ r_{i+1}-r_{i} \text { odd }}} \operatorname{det}_{\substack{1 \leq i, j \leq n \\ \text { odd }}}\left(u_{i}^{r_{j-1}}\right)
$$

Note that as before we have relaxed the condition $r_{0}=0$ into $r_{0} \geq 0$, with $r_{0}$ even (we have introduced $r_{-1} \equiv-1$, and the condition $r_{i+1}-r_{i}$ odd applies for $\left.i=-1,0, \ldots, n-2\right)$. 
The last sum is easily evaluated from a standard result for the sum over all Schur functions corresponding to even partitions (see eq. 4.29 of [田]), namely that

$$
\sum_{\substack{0 \leq r_{0}<r_{1}<\cdots<r_{n-1} \\ r_{i+1}-r_{i} \text { odd }}} \operatorname{det}_{\substack{1 \leq i, j \leq n \\ 1 \leq i<j \leq n}}\left(u_{i}^{r_{j-1}}\right)=\prod_{1-u_{i} u_{j}} \prod_{i=1}^{n} \frac{1}{1-u_{i}^{2}}
$$

and eq.(2.7) follows.

The modified TSSCPP generating polynomial $N_{10}^{\prime}\left(t_{0}, t_{1}, \ldots, t_{n-1}\right)$ will be specialized for later purpose to $t_{0} \equiv t$ and $t_{1}=t_{2}=\cdots=t_{n-1}=\tau$, and we denote by $N_{10}^{\prime}(2 n \mid t, \tau)$ the corresponding polynomial. For instance in the case $n=3, N_{10}^{\prime}(6 \mid t, \tau)=N_{10}^{\prime}(t, \tau, \tau)=$ $t+\tau+2 t^{2} \tau+2 t \tau^{2}+\tau^{3}$. From the remark above, we have $N_{10}^{\prime}(2 n \mid 1, \tau)=N_{10}(2 n \mid \tau)$, as the modified TSSCPPs are in bijection with TSSCPPs, and $t=1$ corresponds to putting no extra weight for the modification. Hence for $\tau=1$, we have $N_{10}^{\prime}(2 n \mid 1,1)=N_{10}(2 n \mid 1)=$ $A_{n}$. This yields for instance $N_{10}^{\prime}(6 \mid 1,1)=\left(t+\tau+2 t^{2} \tau+2 t \tau^{2}+\tau^{3}\right)_{t=\tau=1}=7=A_{3}$.

Interestingly, if we take $t_{0}=0$, the only contributing modified TSSCPPs are those with only diagonal steps in their last row, which enforces the rule that consecutive arrival points on the underlying TSSCPPs (with top slice removed) must have odd integer differences. Therefore

$$
N_{10}^{\prime}\left(t_{0}=0, t_{1}, \ldots, t_{n-1}\right)=N_{10}^{\prime}\left(t_{1}, \ldots, t_{n-1}\right)
$$

Once expressed at the above specialization, this gives $N_{10}^{\prime}(2 n \mid 0, \tau)=N_{10}^{\prime}(2 n-2 \mid \tau, \tau)$. In particular, if we take $\tau=1$, we get $N_{10}^{\prime}(2 n \mid 0,1)=N_{10}^{\prime}(2 n-2 \mid 1,1)=N_{10}(2 n-2 \mid 1)=A_{n-1}$. For instance, $N_{10}^{\prime}(6 \mid 0,1)=\left.\left(t+\tau+2 t^{2} \tau+2 t \tau^{2}+\tau^{3}\right)\right|_{t=0, \tau=1}=2=A_{2}$. Another simple specialization is when $t_{0} \rightarrow \infty$, in which case we retain only the configurations with only vertical steps in the extra slice, hence again such that the endpoints $\left(r_{i}, 0\right)$ of the corresponding TSSCPPs themselves satisfy the parity condition that all $r_{i+1}-r_{i}$ are odd integers:

$$
\lim _{t_{0} \rightarrow \infty} t_{0}^{-(n-1)} N_{10}^{\prime}\left(t_{0}, t_{1}, \ldots, t_{n-1}\right)=N_{10}^{\prime}\left(t_{1}, \ldots, t_{n-1}\right)
$$

When all $t_{i}=1$ for $i=1,2 \ldots, n-1$, this is nothing but $N_{10}^{\prime}(2 n-2 \mid 1,1)=A_{n-1}$. In the case $n=3$, this reduces to $\left.\left.\left(t+\tau+2 t^{2} \tau+2 t \tau^{2}+\tau^{3}\right)\right|_{t^{2}}\right|_{\tau=1}=2=A_{2}$.

The two specializations at $t_{0}=0$ and $t_{0}=1$ will correspond precisely to the cases (ii) and (iii) described in the introduction. For $t$ arbitrary and $\tau=1$, we also get a refinement of the TSSCPP numbers in the form of a polynomial $N_{10}^{\prime}(2 n \mid t, 1)$. The coefficients of this polynomial seem to be the refined alternating sign matrix numbers $A_{n, k}$ that count 
alternating sign matrices of size $n \times n$, with a 1 in position $k$ on their top row. So we have the conjecture that:

$$
N_{10}^{\prime}(2 n \mid t, 1)=\sum_{k=1}^{n} A_{n, k} t^{k-1}
$$

Note that an analogous statement [14] exists for refined TSSCPPs of size $2 n$ according to the steps in their top slice, namely

$$
N_{10}(t, 1,1, \ldots, 1)=\sum_{k=1}^{n} A_{n, k} t^{k-1}
$$

\section{The minimal polynomial solution to the $q \mathrm{KZ}$ equation}

\subsection{Level 1 qKZ equation}

The $q \mathrm{KZ}$ equation was introduced in the context of affine quantum groups [9] and in connection to two-dimensional integrable lattice models [16]. Our basic observation [11] is that it allows for generalizing the groundstate eigenvector condition (1.2) to generic values of $q$. Here we concentrate on the level $1 U_{q}(\widehat{\mathfrak{s l}}) q \mathrm{KZ}$ equation, and refer to [11] for higher rank generalizations. The latter is based on the Temperley-Lieb algebra $T L_{2 n}(\tau)$ with generators $e_{i}, i=1, \ldots, 2 n$ subject to the relations: $e_{i}^{2}=\tau e_{i}$ and $e_{i} e_{i \pm 1} e_{i}=e_{i}$ for all $i$, with the convention that $e_{2 n+1} \equiv e_{1}$. The standard representation of $T L_{2 n}(\tau)$ is on link patterns $\pi \in \mathcal{L}_{2 n}$. The generator $e_{i}$ simply acts by connecting the two points $i, i+1$, and

gluing the two other ends of arches formerly attached to them. If $i$ and $i+1$ were already connected, $e_{i}$ acts as the identity times the parameter $\tau$, which accounts for a weight for the loop thus created. This weight per loop is parametrized as

$$
\tau=-q-q^{-1}
$$

where we might interpret $-q^{\epsilon}$ as a weight per oriented loop, $\epsilon= \pm 1$ according to the orientation, and the weight $\tau$ is obtained by summing over the two possible orientations of each loop.

Using the Temperley-Lieb algebra generators, it is a simple exercise to construct a solution of the unitarity and Yang-Baxter equations, in the form of the quantum $R$-matrix:

$$
\check{R}_{i, i+1}(z, w)=\frac{q z-q^{-1} w}{q w-q^{-1} z} \operatorname{Id}+\frac{z-w}{q w-q^{-1} z} e_{i}
$$


The $q \mathrm{KZ}$ equation reduces to the following relations:

$$
\begin{aligned}
\check{R}_{i, i+1}\left(z_{i+1}, z_{i}\right) \Psi\left(z_{1}, \ldots, z_{2 n}\right) & =\tau_{i} \Psi\left(z_{1}, \ldots, z_{2 n}\right), \quad i=1,2, \ldots, 2 n-1 \\
\sigma \Psi\left(z_{2}, \ldots, z_{2 n}, s z_{1}\right) & =c_{n} \Psi\left(z_{1}, \ldots, z_{2 n}\right)
\end{aligned}
$$

where the operator $\tau_{i}$ acts as the elementary transposition of spectral parameters $z_{i} \leftrightarrow z_{i+1}$ and the operator $\sigma$ is a shift operator $\sigma e_{i} \sigma^{-1}=e_{i+1}$ for all $i$, while the constants $s$ and $c_{n}$ are fixed to be respectively $s=q^{6}$ and $c_{n}=q^{3(n-1)}$.

Expressing [3.3] in the link pattern basis, we get a system of equations determining the components $\Psi_{\pi}$ of the vector $\Psi_{n}$, namely:

$$
\begin{aligned}
\frac{q^{-1} z_{i+1}-q z_{i}}{z_{i+1}-z_{i}}\left(\tau_{i}-1\right) \Psi_{\pi}\left(z_{1}, \ldots, z_{2 n}\right) & =\sum_{\substack{\pi^{\prime} \neq \pi \\
e_{i} \pi^{\prime}=\pi}} \Psi_{\pi^{\prime}}\left(z_{1}, \ldots, z_{2 n}\right) \\
\Psi_{\sigma(\pi)}\left(z_{2}, \ldots, z_{2 n}, q^{6} z_{1}\right) & =q^{3(n-1)} \Psi_{\pi}\left(z_{1}, \ldots, z_{2 n}\right)
\end{aligned}
$$

In [10,12], it was shown that the space, called $V$ in what follows, which is spanned by the $\Psi_{\pi}$ could be simply characterized by the following vanishing condition (also called wheel condition), that any polynomial in that space vanishes whenever any triple of cyclically ordered spectral parameters take values $\left(z, q^{2} z, q^{4} z\right)$. We will make use of this remark below.

Let us quote the explicit form of the base component $\Psi_{\pi_{0}}$ corresponding to the link pattern $\pi_{0}$ that connects $i$ and $N+1-i$ :

$$
\Psi_{\pi_{0}}=\prod_{1 \leq i<j \leq n}\left(q z_{i}-q^{-1} z_{j}\right) \prod_{n+1 \leq i<j \leq N}\left(q z_{i}-q^{-1} z_{j}\right)
$$

as well as a useful property that allows to generate $\Psi_{n}$ recursively:

The components of $\Psi_{n}$, polynomial solution of level $1 q K Z$ equation of degree $n(n-1)$, normalized by Eq. (3.5), satisfy the following recurrence relations:

$$
\left.\Psi_{\pi}\right|_{z_{i+1}=q^{2} z_{i}}= \begin{cases}0 & \text { if } \pi(i) \neq i+1 \\ \prod_{j=1}^{i-1}\left(z_{i}-q^{2} z_{j}\right) \prod_{j=i+2}^{N}\left(q^{2} z_{i}-q^{-2} z_{j}\right) \times & \text { if } \pi(i)=i+1 . \\ \times \Psi_{\pi^{\prime}}\left(z_{1}, \ldots, z_{i-1}, z_{i+2}, \ldots, z_{N}\right) & \end{cases}
$$

where in the second case, $\pi^{\prime}$ is obtained from $\pi$ by deleting the arch $(i, i+1)$. 


\subsection{Integral formulae for the span of solutions}

We now turn to the derivation of multiple residue integral formulas for vectors in the space spanned by the components $\Psi_{\pi}$ of the minimal polynomial solution to the $q \mathrm{KZ}$ equation.

Let $z_{1}, \ldots, z_{N}$ be $N=2 n$ complex indeterminates (spectral parameters). We consider the following multiple contour integrals:

$$
\begin{aligned}
\Psi_{a_{1}, \ldots, a_{n}} & =\prod_{1 \leq i<j \leq N}\left(q z_{i}-q^{-1} z_{j}\right) \times \\
& \times \oint \cdots \oint \prod_{\ell=1}^{n} \frac{\mathrm{d} w_{\ell}}{2 \pi i} \frac{\prod_{1 \leq \ell<m \leq n}\left(w_{m}-w_{\ell}\right)\left(q w_{\ell}-q^{-1} w_{m}\right)}{\prod_{\ell=1}^{n} \prod_{1 \leq i \leq a_{\ell}}\left(w_{\ell}-z_{i}\right) \prod_{a_{\ell}<i \leq N}\left(q w_{\ell}-q^{-1} z_{i}\right)}
\end{aligned}
$$

where $\left(a_{\ell}\right)_{\ell=1, \ldots, n}$ is any non-decreasing sequence of integers in $\{1, \ldots, N-1\}$. The contours catch the poles at $w_{i}=z_{j}$ but not those at $w_{i}=q^{-2} z_{j}$. These integrals are closely related to formulae for solutions of level $1 q \mathrm{KZ}$ equation in the spin basis, as given in e.g. [17]. In appendix B, a detailed discussion of the connection between the two types of integrals is given.

We want to show the following: $\Psi_{a_{1}, \ldots, a_{n}}$ is a homogeneous polynomial in the variables $z_{1}, \ldots, z_{N}$ of degree $n(n-1)$. Furthermore it satisfies the wheel condition: for all ordered triplets $i, j, k$,

$$
\Psi_{a_{1}, \ldots, a_{n}}\left(\ldots, z_{i}=z, \ldots, z_{j}=q^{2} z, \ldots, z_{k}=q^{4} z, \ldots\right)=0 \quad 1 \leq i<j<k \leq N
$$

To prove this, we first write explicitly the residue formula for Eq. [(3.7):

$$
\begin{aligned}
& \Psi_{a_{1}, \ldots, a_{n}}=\prod_{1 \leq i<j \leq N}\left(q z_{i}-q^{-1} z_{j}\right) \times \\
& \times \sum_{\substack{\left\{k_{1}, \ldots, k_{n}\right\} \\
k_{\ell} \neq k_{m}, 1 \leq k_{\ell} \leq a_{\ell}}} \frac{\prod_{1 \leq \ell<m \leq n}\left(z_{k_{m}}-z_{k_{\ell}}\right)\left(q z_{k_{\ell}}-q^{-1} z_{k_{m}}\right)}{\prod_{\ell=1}^{n} \prod_{1 \leq i \leq a_{\ell}, i \neq k_{\ell}}\left(z_{k_{\ell}}-z_{i}\right) \prod_{a_{\ell}<i \leq N}\left(q z_{k_{\ell}}-q^{-1} z_{i}\right)} \\
& =\sum_{\substack{K=\left\{k_{1}, \ldots, k_{n}\right\} \\
k_{\ell} \neq k_{m}, 1 \leq k_{\ell} \leq a_{\ell}}}(-1)^{s\left(k_{\ell}\right)} \frac{\prod_{1 \leq \ell<m \leq n}\left(q z_{k_{\ell}}-q^{-1} z_{k_{m}}\right)}{\prod_{\substack{1 \leq i<j \leq N \\
i \notin K \text { or } i=k_{\ell}, j \leq a_{\ell}}}\left(q z_{i}-q^{-1} z_{j}\right)}
\end{aligned}
$$


where $(-1)^{s\left(k_{\ell}\right)}$ is the sign of the permutation that places the $k_{\ell}$ in increasing order.

Let us now compute the residue at $z_{i} \rightarrow z_{j}$. Note that at least one of the two integers $i, j$ must belong to $K$ for the residue of the summand to be non-zero. Two cases arise: a) terms where both $i$ and $j$ are in $K$, say $j=k_{\ell}$ and $i=k_{m}$ with $k_{\ell}<k_{m} \leq a_{\ell}$ (and as always $\left.k_{m} \leq a_{m}\right)$. Then one can switch $k_{\ell}$ and $k_{m}$ : now $k_{\ell}=i, k_{m}=j, k_{m}<k_{\ell} \leq a_{m}$ so this new term also has a pole at $z_{i} \rightarrow z_{j}$, the residue being the same but with the sign changed (due to $s\left(k_{\ell}\right)$ ). So the two terms cancel. b) terms where only say $j=k_{\ell}$, and $i \notin K$, with $i \leq a_{\ell}$. Consider the term where $k_{\ell}=j$ is replaced with $i$ : again it is tedious but easy to check that it has the same residue with the opposite sign.

$\Psi_{a_{1}, \ldots, a_{n}}$, being a rational fraction without poles, is a polynomial. The homogeneity and degree follow immediately. Consider now $1 \leq i<j<k \leq N$, and $z_{i}=z, z_{j}=q^{2} z$, $z_{k}=q^{4} z$. For each term in the sum of Eq. (3.9): for the second type of factors in the numerator to be non-zero, necessarily $i \in K$ and $j \in K$. Furthermore, for the first type of factors to be non-zero, $i=k_{m}$ and $j=k_{\ell}$ with $\ell<m$. But then $j=k_{\ell} \leq a_{\ell} \leq a_{m}$ (this is where we use the fact that $\left(a_{\ell}\right)$ is non-decreasing), so that the second factor with indices $i, j$ vanishes. All terms vanishing, the sum is zero.

ExAmPLE. For $\Psi_{a_{1}, \ldots, a_{n}}$ to be non-zero, according to the residue formula (3.9), one must have $a_{\ell} \geq \ell$ (in fact, $\ell \leq a_{\ell}<\ell+n$ ). Let us compute the first non-trivial component, that is $\Psi_{1,2, \ldots, n}$. There is a single residue, at $w_{i}=z_{i}, i=1, \ldots, n$, and we obtain the "base" component $\Psi_{\pi_{0}}$ given by Eq. (3.5).

The precise statement found in [10] is that the space $V$ of homogeneous polynomials of degree $n(n-1)$ in the variables $z_{1}, \ldots, z_{N}$ that satisfy the condition [3.8] is of dimension $c_{n}=(2 n) ! / n ! /(n+1)$ !, and that the components $\Psi_{\pi}$ of the level 1 solution of $q \mathrm{KZ}$ equation in the link pattern basis form a basis of this space (the components in the spin basis also span this space, but they are not linearly independent since there are $\left(\begin{array}{c}2 n \\ n\end{array}\right)$ of them). Furthermore, we have the following proposition: a polynomial $P \in V$ is entirely determined by its values at the following $c_{n}$ points, indexed by link patterns $\pi$ :

$$
z_{i}=q^{-\epsilon_{i}(\pi)} \quad \epsilon_{i}(\pi)=\operatorname{sign}(\pi(i)-i)= \begin{cases}+1 & \text { if } \pi \text { has an opening at } i \\ -1 & \text { if } \pi \text { has a closing at } i\end{cases}
$$

Proof. Write $P=\sum_{\pi} a_{\pi} \Psi_{\pi}$, and note that $\Psi_{\pi^{\prime}}\left(q^{-\epsilon_{i}(\pi)}\right) \neq 0$ iff $\pi=\pi^{\prime}$. This is easily proved by induction using Eq. (3.6). Indeed pick any "little arch" of $\pi$, that is $i$ such that $\pi(i)=i+1$. Either a) $\pi^{\prime}(i) \neq i+1$, in which case $\Psi_{\pi^{\prime}}\left(q^{-\epsilon_{i}(\pi)}\right)=0$ (first case of 
Eq. [3.6]); or b) $\pi(i)=i+1$, in which case we may apply the second case of Eq. [3.6] and use the induction hypothesis to conclude. The induction even allows to compute $C_{\pi}:=\Psi_{\pi}\left(q^{-\epsilon_{i}(\pi)}\right)$, though we shall not need these explicit expressions:

$$
C_{\pi}=C \tau^{|\pi|} \quad C=\left(q-q^{-1}\right)^{n(n-1)} \quad|\pi|=n^{2}+\sum_{i=1}^{N} i \epsilon_{i}(\pi)
$$

Going back to $P$, we find that $a_{\pi}=P\left(q^{-\epsilon_{i}(\pi)}\right) / C_{\pi}$, and in particular that $P$ is entirely determined by these values.

Each $\Psi_{a_{1}, \ldots, a_{n}}$ is thus a linear combination of the $\Psi_{\pi}$, and the coefficients are given by a simple evaluation. In the course of this proof we have found that the evaluation of the $\Psi_{\pi}$ themselves is most easily obtained by using recurrence relations; it is therefore natural to try to do the same for the $\Psi_{a_{1}, \ldots, a_{n}}$ :

Assume that $\left(a_{i}\right)$ is an increasing sequence. Then

$$
\left.\Psi_{a_{1}, \ldots, a_{n}}\right|_{z_{i+1}=q^{2} z_{i}}= \begin{cases}0 & \text { if } i \notin\left\{a_{\ell}\right\} . \\ \prod_{j=1}^{i-1}\left(z_{i}-q^{2} z_{j}\right) \prod_{j=i+2}^{N}\left(q^{2} z_{i}-q^{-2} z_{j}\right) \times & \text { if } i=a_{\ell} . \\ \times \Psi_{a_{1}, \ldots, a_{\ell-1}, a_{\ell+1}-2, \ldots, a_{n}-2}\left(z_{1}, \ldots, z_{i-1}, z_{i+2}, \ldots, z_{N}\right)\end{cases}
$$

Note that the sequence $\left(a_{1}, \ldots, a_{\ell-1}, a_{\ell+1}-2, \ldots, a_{n}-2\right)$ is not necessarily increasing (only non-decreasing).

Proof. Assume $z_{i+1}=q^{2} z_{i}$. According to Eq. (3.9), for the second type of factor in the numerator to be non-zero, $i \in K$, so that $i=k_{\ell}$ for some $\ell$, and $i+1>a_{\ell}$. Since $k_{\ell} \leq a_{\ell}, i=k_{\ell}=a_{\ell}$. This proves the first case of Eq. (3.12). For the second case, we see that the $\ell$ above being unique, only the residue at $w_{\ell}=z_{i}$ contributes, so that we can go back to Eq. [3.7] and perform the integration over $w_{\ell}$. We find:

$$
\begin{aligned}
& \Psi_{a_{1}, \ldots, a_{n}}=\prod_{\substack{1 \leq j<k \leq N,(j, k) \neq(i, i+1) \\
\prod_{1 \leq m<m^{\prime} \leq n}\left(w_{m^{\prime}}-w_{m}\right)\left(q w_{m}-q^{-1} w_{m^{\prime}}\right)}}\left(q z_{j}-q^{-1} z_{k}\right) \oint \cdots \oint \prod_{1 \leq m \leq n, m \neq \ell} \frac{\mathrm{d} w_{m}}{2 \pi i} \\
& \frac{\prod_{1 \leq m \leq n, m \neq \ell} \prod_{1 \leq j \leq a_{m}}\left(w_{m}-z_{j}\right) \prod_{a_{m}<j \leq N}\left(q w_{m}-q^{-1} z_{j}\right)}{\prod_{1 \leq m<\ell}\left(z_{i}-w_{m}\right)\left(q w_{m}-q^{-1} z_{i}\right) \prod_{\ell<m \leq n}\left(w_{m}-z_{i}\right)\left(q z_{i}-q^{-1} w_{m}\right)} \\
& \prod_{1 \leq j<i}\left(z_{i}-z_{j}\right) \prod_{i+1<j \leq N}\left(q z_{i}-q^{-1} z_{j}\right)
\end{aligned}
$$

Using $z_{i+1}=q^{2} z_{i}$ results in multiple cancellations, and after pulling out the factors appearing in $\Psi_{a_{1}, \ldots, a_{\ell-1}, a_{\ell+1}-2, \ldots, a_{n}-2}$ with appropriately reindexed variables, we get Eq. (3.12).

This proposition may be generalized to arbitrary sequences, see appendix A. 


\subsection{Partial sums in the link pattern basis}

We now consider a special class of such integrals, corresponding to the following increasing sequences $\left(a_{\ell}\right)$ :

$$
\mathcal{A}_{n}=\left\{\left(a_{\ell}\right)_{1 \leq \ell \leq n}: a_{\ell} \in\{2 \ell-2,2 \ell-1\}\right\}
$$

This defines $2^{n-1}$ different sequences $\left(a_{1}=1\right.$; one could consider $a_{1}=0$ but it would correspond to $\left.\Psi_{0, a_{2}, \ldots, a_{n}}=0\right)$. One interesting property of these is that the recurrence relation (3.12) expresses sequences from $\mathcal{A}_{n}$ in terms of sequences from $\mathcal{A}_{n-1}$. We also consider a partition of the set of link patterns into subsets indexed by the same sequences $\left(a_{\ell}\right) \in \mathcal{A}_{n}:$

$$
\mathcal{L}\left(a_{1}, \ldots, a_{n}\right)=\left\{\pi \in \mathcal{L}_{2 n}: \pi(2 m-1)>2 m-1 \text { iff } 2 m-1 \in\left\{a_{\ell}\right\}, m=1, \ldots, n\right\}
$$

i.e. the set of link patterns whose arch openings on odd sites are exactly the odd elements of the corresponding sequence.

We can now state the following identities:

$$
\Psi_{a_{1}, \ldots, a_{n}}=\sum_{\pi \in \mathcal{L}\left(a_{1}, \ldots, a_{n}\right)} \Psi_{\pi} \quad \forall\left(a_{\ell}\right) \in \mathcal{A}_{n}
$$

Proof. Both sides of Eq. (3.16) belong to $V$. It thus suffices to show that they are equal at the $c_{n}$ values of Eq. (3.10). To perform this evaluation for the l.h.s. it is enough to use Eq. (3.12), i.e. use recurrence relations at $z_{i+1}=q^{2} z_{i}$ : indeed, to evaluate at $z_{i}=q^{-\epsilon_{i}(\pi)}$, one can pick a little $\operatorname{arch}(i, i+1)$ of $\pi$ and note that $z_{i+1}=q=q^{2} \times q^{-1}=q^{2} z_{i}$, so that one can apply the recurrence relation, which reduces to the same evaluation (with link pattern obtained from $\pi$ by removal of the little $\operatorname{arch}(i, i+1))$ in size $n-1$. Thus, one must show the same recurrence relations for the r.h.s. We shall use Eq. (3.6). Set $z_{i+1}=q^{2} z_{i}$, and assume first $i \notin\left\{a_{\ell}\right\}$. If $i$ is odd that means all $\pi \in \mathcal{L}\left(a_{\ell}\right)$ do not have an opening at $i$. If $i$ is even then $i+1 \in\left\{a_{\ell}\right\}$ (cf Eq. (3.14) ) and thus all $\pi \in \mathcal{L}\left(a_{\ell}\right)$ have an opening at $i+1$. In both cases we conclude that they have no little arch between $i$ and $i+1$ and thus are zero, so that the r.h.s. of Eq. (3.16) is zero. Assume now $i=a_{\ell}$. Among the $\pi \in \mathcal{L}\left(a_{1}, \ldots, a_{n}\right)$, there are two categories: those with $\pi(i) \neq i+1$, which do not contribute to the sum at $z_{i+1}=q^{2} z_{i}$; and those with $\pi(i)=i+1$. It is easy to check that the latter are in bijection with the $\pi^{\prime} \in \mathcal{L}\left(a_{1}, \ldots, a_{\ell-1}, a_{\ell+1}-2, \ldots, a_{n}-2\right)$, the bijection consisting as usual in the deletion of the little arch $(i, i+1)$. Since the prefactors in Eqs. [3.6) and [3.12) are the same, we conclude that the recurrence relations satisfied by both sides of Eq. [3.12] are identical. The initial condition at $n=0$ is also identical: $\Psi_{\emptyset}=1$. 
Example. At $n=3, \mathcal{A}_{3}=\{(1,3,5),(1,3,4),(1,2,5),(1,2,4)\}$ and the partition of link patterns is



Note that $\mathcal{L}(1,3, \ldots, 2 n-1)$ is always the singlet $\pi(2 \ell-1)=2 \ell, \ell=1, \ldots, n$ and that $\mathcal{L}(1,2, \ldots, 2 n-2)$ is always the singlet $\pi(1)=N, \pi(2 \ell)=2 \ell+1, \ell=1, \ldots, n-1$. In general, $\mathcal{L}\left(a_{1}, \ldots, a_{n}\right)$ contains the link pattern whose openings are exactly the $a_{\ell}$, but may have more elements.

\subsection{Homogeneous limit}

Finally we consider the homogeneous limit $z_{1}=\cdots=z_{2 n}=1$; We rewrite Eq. (3.7) with this specialization and normalize it with $\Psi_{\pi_{0}}=\left(q-q^{-1}\right)^{n(n-1)}$ :

$$
\Psi_{a_{1}, \ldots, a_{n}} / \Psi_{\pi_{0}}=\left(q-q^{-1}\right)^{n^{2}} \oint \ldots \oint \prod_{\ell=1}^{n} \frac{\mathrm{d} w_{\ell}}{2 \pi i} \frac{\prod_{1 \leq \ell<m \leq n}\left(w_{m}-w_{\ell}\right)\left(q w_{\ell}-q^{-1} w_{m}\right)}{\prod_{\ell=1}^{n}\left(w_{\ell}-1\right)^{a_{\ell}}\left(q w_{\ell}-q^{-1}\right)^{2 n-a_{\ell}}}
$$

Changing variables: $w_{\ell}=\frac{1-q^{-1} u_{\ell}}{1-q u_{\ell}}$ results in

$$
\Psi_{a_{1}, \ldots, a_{n}} / \Psi_{\pi_{0}}=\oint \cdots \oint \prod_{\ell=1}^{n} \frac{\mathrm{d} u_{\ell}}{2 \pi i u_{\ell}^{a_{\ell}}} \prod_{1 \leq \ell<m \leq n}\left(u_{m}-u_{\ell}\right)\left(1+u_{\ell} u_{m}+\tau u_{m}\right)
$$

where the contours surround 0 , and $\tau=-q-q^{-1}$.

As a corollary, the sum of all components is

$$
\sum_{\pi \in \mathcal{L}_{2 n}} \Psi_{\pi} / \Psi_{\pi_{0}}=\oint \cdots \oint \prod_{\ell=1}^{n} \frac{\left(1+u_{\ell}\right) \mathrm{d} u_{\ell}}{2 \pi i u_{\ell}^{2 \ell-1}} \prod_{1 \leq \ell<m \leq n}\left(u_{m}-u_{\ell}\right)\left(1+u_{\ell} u_{m}+\tau u_{m}\right)
$$

This is obtained by summing Eq. (3.18) over all sequences $\left(a_{\ell}\right) \in \mathcal{A}_{n}$ and applying Eq. (3.16).

ExAmple. At $n=3, \Psi_{1,3,5}=\tau^{3}+\tau, \Psi_{1,3,4}=\tau^{2}+1, \Psi_{1,2,5}=\tau^{2}, \Psi_{1,2,4}=2 \tau$ and the full sum is $\sum_{\pi} \Psi_{\pi}=\tau^{3}+2 \tau^{2}+3 \tau+1$. 


\section{From integral formulae back to TSSCPPs}

\subsection{A refined $q K Z-T S S C P P$ conjecture}

Let us consider once again the generating series $N_{10}^{\prime}$ of modified TSSCPPs specialized to $t_{0}=t$ and $t_{1}=t_{2}=\cdots=t_{n-1}=\tau$ : according to Eq. (2.7), it is given by

$$
N_{10}^{\prime}(2 n \mid t, \tau)=\oint \cdots \oint \prod_{\ell=1}^{n} \frac{\mathrm{d} u_{\ell}\left(1+t u_{\ell}\right)\left(1+\tau u_{\ell}\right)^{\ell-1}}{2 \pi i u_{\ell}^{2 \ell-1}} \frac{\prod_{1 \leq \ell<m \leq n}\left(u_{m}-u_{\ell}\right)}{\prod_{1 \leq \ell \leq m \leq n}\left(1-u_{\ell} u_{m}\right)}
$$

On the other hand, in view of the partial sums of Eq. (3.16), it is natural to define the following generating series:

$$
\hat{N}_{10}^{\prime}(2 n \mid t, \tau):=\sum_{\left(a_{\ell}\right) \in A} t^{\sum_{\ell}\left(2 \ell-1-a_{\ell}\right)} \sum_{\pi \in \mathcal{L}\left(a_{1}, \ldots, a_{n}\right)} \Psi_{\pi} / \Psi_{\pi_{0}}
$$

Applying Eq. (3.18), we find the following formula:

$$
\hat{N}_{10}^{\prime}(2 n \mid t, \tau)=\oint \cdots \oint \prod_{\ell=1}^{n} \frac{\mathrm{d} u_{\ell}\left(1+t u_{\ell}\right)}{2 \pi i u_{\ell}^{2 \ell-1}} \prod_{1 \leq \ell<m \leq n}\left(u_{m}-u_{\ell}\right)\left(1+\tau u_{m}+u_{\ell} u_{m}\right)
$$

Note that at $t=1 \hat{N}_{10}^{\prime}(2 n \mid 1, \tau)$ is simply the sum of all components in the loop basis. The content of the TSSCPP conjecture of [1] is thus that $\hat{N}_{10}^{\prime}(2 n \mid 1, \tau)=N_{10}^{\prime}(2 n \mid 1, \tau)$.

Observe further that at $t=0, \hat{N}_{10}^{\prime}(2 n \mid 0, \tau)$ is the largest component $\Psi_{\max } / \Psi_{\pi_{0}}$ whereas $N_{10}^{\prime}(2 n \mid 0, \tau)=N_{10}^{\prime}(2 n-2 \mid \tau, \tau)$. In particular at $\tau=1$ they both equal $A_{n-1}$.

In view of these specializations and of numerical experimentation, we have been led to the conjecture that $N_{10}^{\prime}(2 n \mid t, \tau)=\hat{N}_{10}^{\prime}(2 n \mid t, \tau)$ for all values of the parameters. For example, at $n=3, \hat{N}_{10}^{\prime}(6 \mid t, \tau)=\Psi_{1,3,5}+t\left(\Psi_{1,3,4}+\Psi_{1,2,5}\right)+t^{2} \Psi_{1,2,4}=\tau^{3}+2 t \tau^{2}+2 t^{2} \tau+\tau+t$, which coincides with $N_{10}^{\prime}(6 \mid t, \tau)$ given in Sect. 2 .

\subsection{Attempted proof and a conjectured identity}

In order to show this conjecture, all one needs to do is to prove the equality of Eqs. (4.1) and (4.3). Considering the free fermionic nature of TSSCPPs, it is natural to antisymmetrize these expressions. We find the following formulae:

$$
\begin{aligned}
& \left\{\prod_{1 \leq \ell \leq m \leq n}\left(1-u_{\ell} u_{m}\right) \operatorname{AS}\left(\prod_{i=1}^{n} u_{\ell}^{-2 \ell+2} \prod_{1 \leq \ell<m \leq n}\left(1+u_{\ell} u_{m}+\tau u_{m}\right)\right)\right\}_{\leq 0} \\
& =\operatorname{AS}\left(\prod_{\ell=1}^{n}\left(u_{\ell}^{-1}\left(\tau+u_{\ell}^{-1}\right)\right)^{\ell-1}\right)=\prod_{1 \leq \ell<m \leq n}\left(u_{m}^{-1}-u_{\ell}^{-1}\right)\left(\tau+u_{\ell}^{-1}+u_{m}^{-1}\right)
\end{aligned}
$$


where AS stands for the antisymmetrization with respect to permutations of the $u$ 's, namely $A S\left(f\left(u_{1}, \ldots, u_{n}\right)\right)=\sum_{\sigma \in \mathcal{S}_{n}}(-1)^{\sigma} f\left(u_{\sigma(1)}, \ldots, u_{\sigma(n)}\right)$, and the subscript $\leq 0$ means keeping only terms with negative or zero powers in all the variables $u_{\ell}$. The equality in the second line is elementary, but the antisymmetrization in the first is non-trivial. It is easy to check that Eq. (4.4) implies that the symmetrized integrands of Eqs. (4.1) and [4.3)] are equal, so that the integrals are equal.

Equivalently, we can rewrite Eq. (4.4) as the following integral identity:

$$
\begin{gathered}
\oint \cdots \oint \prod_{\ell=1}^{n} \frac{\mathrm{d} u_{\ell}}{2 \pi i} \frac{1-x u_{\ell}^{2}}{2 \pi i u_{\ell}^{2 \ell}} \operatorname{det}_{1 \leq \ell, m \leq n}\left(\frac{1}{1-\alpha_{\ell} u_{m}}\right) \prod_{1 \leq \ell<m \leq n}\left(1+\tau u_{m}+x u_{\ell} u_{m}\right)\left(1-x u_{\ell} u_{m}\right) \\
=\prod_{\ell=1}^{n} \alpha_{\ell} \prod_{1 \leq \ell<m \leq n}\left(\alpha_{m}-\alpha_{\ell}\right)\left(\tau+\alpha_{\ell}+\alpha_{m}\right)
\end{gathered}
$$

There are two important things about this identity. The determinant in the integrand is, up to a Vandermonde determinant of the $u$ 's, the generating function for Schur polynomials of the $u$ 's, themselves spanning the space of symmetric polynomials of these variables. Moreover, the result is clearly independent of the parameter $x$. Combining these two facts, we see that if we replace the determinant by any symmetric polynomial of the $u$ 's times the Vandermonde determinant of the $u$ 's the integral remains independent of $x$. With an appropriate choice of symmetric function and by equating the $x=1$ and $x=0$ values we recover the equality of Eqs. (4.1) and (4.3).

\subsection{Prospects}

The great similarity of Eqs. (4.1) and (4.3) is extremely suggestive. However these formulae are also reminiscent of those of [18], which means that proving they are equal might be more difficult than it seems. We shall end with a few general comments.

First note that the main conjectured result of this paper, expounded in Sect. 4.1, relates four different kinds of objects: at generic parameter $\tau$, it relates the $\tau$-weighted counting of TSSCPPs with an additional weight $t$ depending on the parity of their endpoints (or equivalently, of augmented TSSCPPs with a special weight for the last line) and the sum of components of the polynomial solution to the level $1 q \mathrm{KZ}$ equation (with $\tau=-q-q^{-1}$ ) in the link pattern basis, with an extra weight $t$ depending on the parity of endpoints of loops. When specialized to $\tau=1$, it also relates these to the $t$-refined enumeration of ASMs and to the sum of components of the ground state of the Temperley-Lieb 
model of loops in which all spectral parameters but one are taken to 1, the latter playing the role of $t$.

None of these connections are fully understood. The introduction of spectral parameters, the main idea of [6], has shed some light on the matter, but it is not obvious how to reintroduce a full set of parameters into the TSSCPP conjectures. The first obvious idea that might come to mind is to use the integrability of the rhombus tiling model, and decorate it with spectral parameters, but our attempts were unsuccessful. Note however that for instance such a connection between the partition function for the inhomogeneous model of rhombus tilings of an hexagon of shape $a \times b \times c$ and the actual components of $\Psi$ corresponding to link patterns with three sets of nested arches was established in [19].

The techniques of the present paper may be generalized to other boundary conditions as well. For instance, it was conjectured in [2] that the sum of components of the solution to the level $1 \mathrm{qKZ}$ equation with open (reflecting) boundaries equates in the homogeneous and generic $q$ limit the generating polynomial for $\tau$-weighted (possibly modified) Cyclically Symmetric Transpose Complementary Plane Partitions (CSTCPP), namely cyclically and reflection-invariant rhombus tilings of a hexagon (possibly with a central triangular hole). More general boundaries were considered in [20], and we believe that integral formulae, analogous to those derived in the present paper, must exist for various sum rules of the components of the polynomial solution $\Psi$ to the corresponding qKZ equations, probably allowing for a relation to $\tau$-enumeration of plane partitions with suitable symmetries. We might also wonder how this may generalize to the higher rank and higher spin generalizations of [11] and [21]. Note however that in the higher rank/spin cases, the generalized RS sum rules found lead to sequences of integer numbers for which no combinatorial interpretation exists yet, and it is an open challenge to find out what generalization of ASM/FPL or TSSCPP they could correspond to.

Finally, one can note the great similarity of the present work with the recent paper 22]. This will be discussed elsewhere 23].

\section{Note added}

After this article was completed, Doron Zeilberger found a proof of Eq. (4.4), which can be found on his web site: http://www. math.rutgers.edu/ zeilberg/pj.html 


\section{Acknowledgments}

The authors acknowledge the support of European Marie Curie Research Training Networks "ENIGMA" MRT-CT-2004-5652, "ENRAGE" MRTN-CT-2004-005616, ESF pro-

gram "MISGAM" and of ANR program "GIMP" ANR-05-BLAN-0029-01. PZJ wishes to thank the organizers of the conference CQIS'07, where this work was completed. 


\section{References}

[1] P. Di Francesco, Totally Symmetric Self-Complementary Plane Partitions and Quantum Knizhnik-Zamolodchikov equation: a conjecture, J. Stat. Mech. (2006) P09008, cond-mat/0607499.

[2] P. Di Francesco, Open boundary Quantum Knizhnik-Zamolodchikov equation and the weighted enumeration of Plane Partitions with symmetries, J. Stat. Mech. (2007) P01024, math-ph/0611012.

[3] M.T. Batchelor, J. de Gier and B. Nienhuis, The quantum symmetric XXZ chain at $\Delta=-1 / 2$, alternating sign matrices and plane partitions, J. Phys. A34 (2001) L265-L270, cond-mat/0101385.

[4] D. Bressoud, Proofs and Confirmations: The Story of the Alternating Sign Matrix Conjecture, Cambridge Univ. Pr., 1999.

[5] A.V. Razumov and Yu.G. Stroganov, Combinatorial nature of ground state vector of O(1) loop model, Theor. Math. Phys. 138 (2004) 333-337; Teor. Mat. Fiz. 138 (2004) 395-400, math.C0/0104216.

[6] P. Di Francesco and P. Zinn-Justin, Around the Razumov-Stroganov conjecture: proof of a multi-parameter sum rule, E. J. Combi. 12 (1) (2005), R6, math-ph/0410061.

[7] A. Izergin, Partition function of the six-vertex model in a finite volume, Sov. Phys. Dokl. 32 (1987) 878-879; V. Korepin, Calculation of norms of Bethe wave functions, Comm. Math. Phys. 86 (1982) 391-418.

[8] G. Kuperberg, Symmetry classes of alternating-sign matrices under one roof, Ann. of Math. (2) 156 (2002), no. 3, 835-866, math.C0/0008184.

[9] I.B. Frenkel and N. Reshetikhin, Quantum affine Algebras and Holonomic Difference Equations, Commun. Math. Phys. 146 (1992), 1-60.

[10] V. Pasquier, Quantum incompressibility and Razumov Stroganov type conjectures, Ann. Henri Poincaré 7 (2006), 397-421, cond-mat/0506075.

[11] P. Di Francesco and P. Zinn-Justin, Quantum Knizhnik-Zamolodchikov equation, generalized Razumov-Stroganov sum rules and extended Joseph polynomials, J. Phys. A 38 (2005) L815-L822, math-ph/0508059.

[12] M. Kasatani and V. Pasquier, On polynomials interpolating between the stationary state of a $O(n)$ model and a Q.H.E. ground state, cond-mat/0608160.

[13] P. Di Francesco, Inhomogeneous loop models with open boundaries, J. Phys. A: Math. Gen. 386091 (2005), math-ph/0504032;

Boundary $q K Z$ equation and generalized Razumov-Stroganov sum rules for open IRF models, J. Stat. Mech. P11003 (2005), math-ph/0509011.

[14] D. Robbins, The story of 1,2,7,42,429,7436,.., Mathl. Intelligencer 13 No.2 (1991) 12-19. 
[15] B. Lindström, On the vector representations of induced matroids, Bull. London Math. Soc. 5 (1973) 85-90;

I. M. Gessel and X. Viennot, Binomial determinants, paths and hook formulae, Adv. Math. 58 (1985) 300-321.

[16] F.A. Smirnov, A general formula for soliton form factors in the quantum sine-Gordon model, J. Phys. A 19 (1986), L575-L578.

[17] M. Jimbo and T. Miwa, Algebraic Analysis of Solvable Lattice Models, CBMS 85, American Mathematical Society (1995).

[18] D. Zeilberger, Proof of the alternating sign matrix conjecture, Elec. J. Comb. 3(2) (1996), R13, math.C0/9407211.

[19] P. Zinn-Justin, Proof of Razumov-Stroganov conjecture for some infinite families of link patterns, math.CO/0607183.

[20] P. Di Francesco and P. Zinn-Justin, From Orbital Varieties to Alternating Sign Matrices, extended abstract for FPSAC'06 (2006), math-ph/0512047.

[21] P. Zinn-Justin, Combinatorial point for higher spin loop models, math-ph/0603018.

[22] A.V. Razumov and Yu.G. Stroganov, Bethe roots and refined enumeration of alternating-sign matrices, J. Stat. Mech. (2006) P07004, math-ph/0605004.

[23] A.V. Razumov, Yu.G. Stroganov and P. Zinn-Justin, work in progress. 


\section{Appendix A. Computing coefficients of the $\Psi_{a_{1}, \ldots, a_{n}}$ in the link pattern basis}

Eq. [3.12), which provides recurrence relations for $\Psi_{a_{1}, \ldots, a_{n}}$ for $\left(a_{\ell}\right)$ an increasing sequence, can in fact be generalized to arbitrary non-decreasing sequences:

$$
\begin{aligned}
& \left.\Psi_{a_{1}, \ldots, \underbrace{}_{k}, \ldots, i, \ldots, a_{n}}\right|_{z_{i+1}=q^{2} z_{i}}=\prod_{j=1}^{i-1}\left(z_{i}-q^{2} z_{j}\right) \prod_{j=i+2}^{N}\left(q^{2} z_{i}-q^{-2} z_{j}\right) \times \\
& \times \frac{q^{k}-q^{-k}}{q-q^{-1}} \Psi_{a_{1}, \ldots, \underbrace{i-1, \ldots, i-1}_{k-1}}, \ldots, a_{n}-2\left(z_{1}, \ldots, z_{i-1}, z_{i+2}, \ldots, z_{N}\right)
\end{aligned}
$$

The proof is along the same lines as that of Eq. [3.12].

As a consequence, if one wants to compute the coefficients of $\Psi_{\pi}$ in the link pattern basis by using evaluation of Eq. [3.10], the use of Eq. (A.1) leads to the following: first the non-zero coefficients correspond to link patterns whose little arches open in $\left\{a_{\ell}\right\}$; for each of them, the computation by recurrence involves removing the (say, leftmost) little arch at each step; the number $k$ marked on the opening determines the coefficient $U_{k-1}=\left(q^{k}-q^{-k}\right) /\left(q-q^{-1}\right)$, and the sum of both numbers on opening and closing minus 1 is added to the site at its left.

It is convenient to describe graphically this process as follows. Shift the $a_{i}$ by one half-step to the right, and put a little marker (red circle) indicating the number of $a_{\ell}$ at that location. The recurrence process is to remove one little arch: if there is no marker inside the contribution is zero; otherwise, substract one to the value $k$ of the marker inside, possibly removing it if it becomes zero, and multiply the contribution by the corresponding $U_{k-1}$.

EXAmple. For $\Psi_{1,3,4,5}$, the non-zero coefficients are:
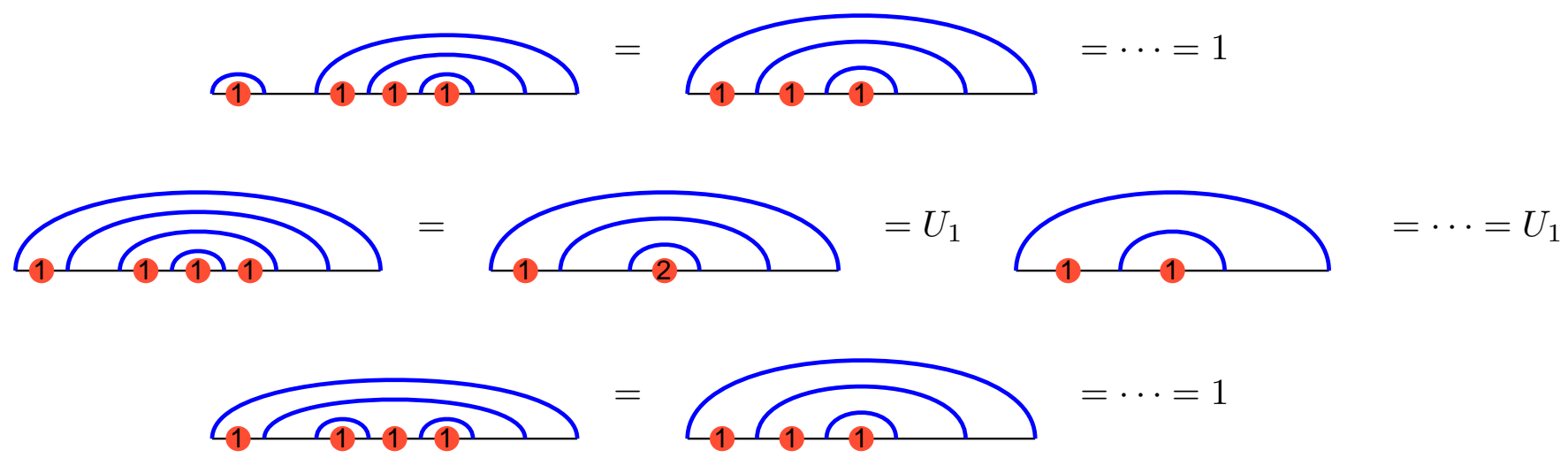

For $\Psi_{2,4,5,5}$

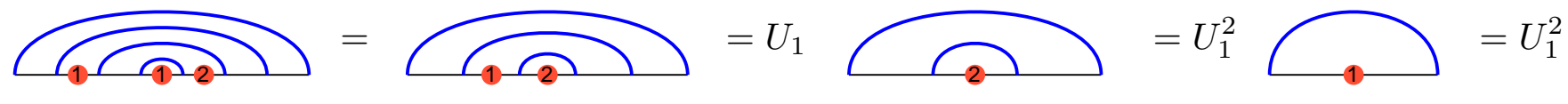






The recurrence can of course be solved, and the final formula for the coefficient of $\Psi_{\pi}$ in $\Psi_{a_{1}, \ldots, a_{n}}$ is:

$$
\prod_{i<\pi(i)} U_{\#\left\{\ell: i \leq a_{\ell}<\pi(i)\right\}-(\pi(i)-i+1) / 2}
$$

where $U_{-1}=0, U_{0}=1, U_{1}=-\tau, U_{2}=\tau^{2}-1$, etc.

As a check, note that this allows to recover Eq. (3.16). Indeed, if $a_{\ell} \in\{2 \ell-2,2 \ell-1\}$, one has

$\#\left\{\ell: i \leq a_{\ell}<\pi(i)\right\}= \begin{cases}(\pi(i)-i+1) / 2 & i \text { even and } \pi(i)-1 \in\left\{a_{\ell}\right\} \text { or } i \text { odd and } i \in\left\{a_{\ell}\right\} \\ (\pi(i)-i-1) / 2 & \text { otherwise. }\end{cases}$

\section{Appendix B. Spin basis components}

The expression of the solution of level $1 q \mathrm{KZ}$ equation in the spin basis (basis of sequences of $n$ +'s and $n$-'s), as given in [17], once rid of various prefactors which are irrelevant for our purposes, reads:

$$
\begin{aligned}
& \tilde{\Psi}_{a_{1}, \ldots, a_{n}}=\left(q-q^{-1}\right)^{n} \prod_{1 \leq i<j \leq N}\left(q z_{i}-q^{-1} z_{j}\right) \\
& \oint \cdots \oint \prod_{\ell=1}^{n} \frac{w_{\ell} \mathrm{d} w_{\ell}}{2 \pi i} \frac{\prod_{1 \leq \ell<m \leq n}\left(w_{m}-w_{\ell}\right)\left(q w_{\ell}-q^{-1} w_{m}\right)}{\prod_{\ell=1}^{n} \prod_{1 \leq i \leq a_{\ell}}\left(w_{\ell}-z_{i}\right) \prod_{a_{\ell} \leq i \leq N}\left(q w_{\ell}-q^{-1} z_{i}\right)}
\end{aligned}
$$

in which the $a_{\ell}$ are the locations of the +'s. Noting that $\left(q-q^{-1}\right) w_{\ell}=\left(q w_{\ell}-q^{-1} z_{i}\right)-$ $q^{-1}\left(w_{\ell}-z_{i}\right)$ and comparing with Eq. (3.7), we find:

$$
\tilde{\Psi}_{a_{1}, \ldots, a_{n}}=\sum_{\varepsilon_{1}, \ldots, \varepsilon_{n} \in\{0,1\}}(-q)^{-\sum_{i} \varepsilon_{i}} \Psi_{a_{1}-\varepsilon_{1}, \ldots, a_{n}-\varepsilon_{n}}
$$

In particular in the homogeneous limit we have the integral formula, analogous to (3.18):

$$
\tilde{\Psi}_{a_{1}, \ldots, a_{n}}=\oint \cdots \oint \prod_{\ell=1}^{n} \frac{\mathrm{d} u_{\ell}\left(1-q^{-1} u_{\ell}\right)}{2 \pi i u_{\ell}^{a_{\ell}}} \prod_{1 \leq \ell<m \leq n}\left(u_{m}-u_{\ell}\right)\left(1+u_{\ell} u_{m}+\tau u_{m}\right)
$$

Note that the formula for $a_{\ell}=2 \ell-1$ ("largest component" in the spin basis $+-\cdots+-$ ) is the special case $t=-q^{-1}$ of the "refined enumeration" of Eq. (4.3).

It is perhaps interesting that one can recover the change of basis from link patterns to spins in our formalism. Indeed, using the method of appendix A we can content ourselves 
with examining the contribution of a little arch $\{i, i+1\}$. The meaning of Eq. [B.1] is that each + can be moved either one half-step to the right with weight 1 , or one half-step to the left with weight $-q^{-1}$. We have four local configurations around $i, i+1$ :


and note that in the last case all contributions cancel, so that we recover the usual rule that an arch gets a weight of 1 if its opening has a + and its closing a - , a weight of $-q^{-1}$ if its opening has a - and its closing a + , and zero otherwise. 